\title{
A new high-integrated weak field sensor for automotive applications
}

\author{
T. Thiessen ${ }^{1}$ and M. Prochaska ${ }^{2}$ \\ ${ }^{1}$ Leibniz Universität Hannover, Institut für Theoretische Elektrotechnik, Appelstr. 9A, 30167 Hannover, Germany \\ ${ }^{2}$ NXP Semiconductors Germany GmbH Automotive Innovation Center Stresemannallee 101, 22529 Hamburg, Germany
}

\begin{abstract}
Especially in the field of automotive applications smart sensor systems for magnetic field sensing face increasing requirements concerning low cost, low power consumption and high magnetic performance. Over the past years AMR sensors play a decisive role in this application area because of their robustness and stability. In the following a high-integrated smart sensor system for magnetic field sensing is presented. A novel approach for the detection of weak magnetic fields is shown, which is based on an AC-excitation of AMR elements. In contrast to common used sensors this concept is based on a nonlinear AMR element without Barber pole construction. By means of this methodology sensitivity as well as temperature and life time stability is significantly improved, while the production costs compared to common-used sensors are reduced. Within this novel approach new signal conditioning algorithms and analog circuit topologies are presented, which are able to meet low offset and low noise requirements.
\end{abstract}

\section{Introduction}

For the measurement of weak magnetic fields various transducer principles are applicable like Fluxgate, Hall, Anisotropic (AMR) or Giant (GMR) Magnetoresistive sensors (Ripka, 2000). However, especially for automotive applications AMR sensors are widely used, because of their good performance concerning temperature stability, high robustness against large magnetic fields and relatively high sensitivity. In addition, usually a linear transfer characteristic, low noise and a negligible hysteresis are demanded. Most AMR sensors, which are commercially available, use a Barber pole construction for the linearization of the transfer

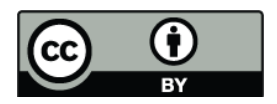

Correspondence to: T. Thiessen (tina.thiessen@gmx.de) function (Ripka, 2000; Kuijk et al., 1975). However, Barber pole sensors need a bias field applied along the easy axis to improve their stability, since the internal magnetization of the ferromagnetic sensor element has two stable positions (Dibbern, 1989). Otherwise, if for any reason a powerful magnetic field appears opposing the internal field, the magnetization flips from one into the opposite direction. Unfortunately, this stabilization field lowers significantly the sensitivity (Tumanski, 2001). So far Barber pole sensors fulfill sensitivity, low noise and hysteresis requirements. However, recent developments especially in the field of automotive applications demand weak field sensing systems which have to satisfy further demands such as low power consumption, low temperature and low life time drift. Typical examples are wheel speed sensing in automotive transmission systems, current measurement or electronic compassing. In addition, the magnetic field strength in automotive systems is often so violent that the measurement range of common-used weak field sensors is inadequately. AC-biasing techniques such as modulation or flipping methods - are well-known to improve the performance of MR sensors (Tumanski, 2001)(Razavi, 1997). However, usually they are not able to fulfill low power as well as low cost requirements of industrial and automotive applications.

In the following a novel approach is presented which is based on a superhet structure of the signal processing unit (Razavi, 1997). The initial point is a nonlinearized AMR element which guarantees a high sensitivity in view of the fact that a bias field along the anisotropic axis is not needed. Since the sinusoidal excitation signal along the hard axis driven by on-chip coils is small, low power requirements are fulfilled. The superhet structure leads to a linearization of the sensor output signal in frequency domain, where hystereses as well as offset effects are eliminated. Since offset effects are mainly responsible for temperature and life time drift this approach also leads to a highly stable sensor characteristic, where the natural robustness against high magnetic fields is

Published by Copernicus Publications on behalf of the URSI Landesausschuss in der Bundesrepublik Deutschland e.V. 


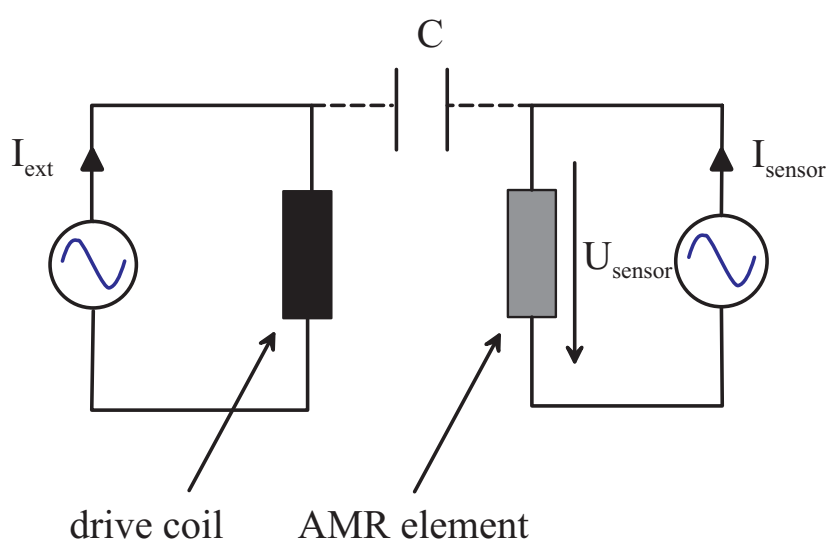

Fig. 1. Simplified circuit diagram of current fed AMR sensor with sinusoidal excitation.

preserved. Furthermore, the elimination of offsets caused by the AMR effect enables in addition with the lock-in character of the sensor signal processing an efficient amplification of output signals of the sensor elements (Meade, 1994).

However, a basic superhet structure consisting of filters and mixer circuits is not able to fulfill low cost demands. Furthermore, AC-driven systems show a capacitive coupling effect between MR layer and coil layer which requires a complex signal processing to separate the speed information from the capacitive coupled frequency. Finally, most AC-driven AMR sensors possess a small air gap range, since large magnetic inputs lead to distortions, which cannot be eliminated by a filter arrangement.

In the following a new method is presented by which the conversion of the speed signal from bandpass to baseband takes place in the AMR element itself and needs no mixer. Coincidentally the new approach separates the speed information from the capacitive coupled frequency. To eliminate the capacitive coupled frequency and reduce the requirements of the signal processing unit a difference principle is used, which leads to significant cost down. Furthermore the method features a large air gap capability, high sensitivity and a high (dynamic) air gap range. The reader should note that the presented methodology is also applicable to other $\mathrm{xMR}$ technologies such as GMR.

\section{Methodology}

As shown in Fig. 1, the invention is based on a nonlinearized AMR element driven by a sinusoidal current and an on-chip coil, which creates a sinusoidal magnetic excitation in ydirection. Between the MR layer and the coil layer occurs a capacitive coupling effect represented by $\mathrm{C}$. The transfer characteristic of a nonlinearized AMR element is given by

$$
R=R_{0}+\Delta R \cos ^{2} \alpha
$$

where $R_{0}$ represents the resistance of the AMR element, $\Delta R$ the maximum change of $R_{0}$ and $\alpha$ with

$$
\sin \alpha=\frac{H_{y}}{H_{x}+H_{0}}
$$

the angle between the internal magnetization of a ferromagnetic thin film and the direction of the current through the film (Dibbern, 1989). $H_{x}$ corresponds to the external magnetic field along the anisotropic axis, $H_{y}$ is the field perpendicular to the easy axis and $H_{0}$ can be regarded as a material constant compromising the so called demagnetizing and anisotropic fields (Tumanski, 2001). For small magnetic fields $H_{y}$ in y-direction and a negligible field in $\mathrm{x}$ direction $\left(H_{x} \rightarrow 0\right)$ AMR sensors without Barber poles can be described by

$$
\begin{aligned}
& R=R_{0}+\Delta R\left(1-\left(\frac{H_{y}}{H_{0}}\right)^{2}\right) \\
& R=R_{0} \text { for } \quad H_{y}>H_{0}
\end{aligned}
$$

If we generate by means of an on-chip coil a sinusoidal modulation field $H_{\mathrm{exc}}(t)$ and assume without a loss of generality an external sinusoidal field $H_{\text {ext }}(t)$, it follows

$H_{y}=\hat{H}_{\mathrm{ext}} \sin \left(\omega_{\mathrm{ext}} t\right)+\hat{H}_{\mathrm{exc}} \sin \left(\omega_{\mathrm{exc}} t\right)$

where $\omega_{\mathrm{ext}}=2 \pi f_{\mathrm{ext}}$ and $\omega_{\mathrm{exc}}=2 \pi f_{\mathrm{exc}}$. Sinusoidal fields $H_{\mathrm{ext}}$ are typical in the field of wheel speed sensing, e.g. anti lock braking systems (ABS) or automotive transmissions, where the amplitude can vary caused by mechanical torsions up to the factor 8 .

If we put Eq. (4) into Eq. (3) we arrive at

$$
\begin{aligned}
& R_{\text {sensor }}(t)=R_{0}+\Delta R\left(1-\frac{\hat{H}_{\mathrm{ext}}^{2}}{2 H_{0}^{2}}-\frac{\hat{H}_{\mathrm{exc}}^{2}}{2 H_{0}^{2}}\right)+\cdots \\
& +\Delta R \frac{\hat{H}_{\mathrm{ext}}^{2}}{2 H_{0}^{2}} \cos \left(2 \omega_{\mathrm{ext}} t\right)+\Delta R \frac{\hat{H}_{\mathrm{exc}}^{2}}{2 H_{0}^{2}} \cos \left(2 \omega_{\mathrm{exc}} t\right)-\cdots \\
& -\Delta R \frac{\hat{H}_{\mathrm{ext}} \hat{H}_{\mathrm{exc}}}{H_{0}^{2}}\left(\cos \left(\left(\omega_{\mathrm{exc}}-\omega_{\mathrm{ext}}\right) t\right)-\cdots\right. \\
& \left.-\cos \left(\left(\omega_{\mathrm{exc}}+\omega_{\mathrm{ext}}\right) t\right)\right),
\end{aligned}
$$

which represents a amplitude modulation of the external signal $H_{\text {exc }}(t)$ by $H_{\text {ext }}(t)$. First, we assume, that the output voltage of a current fed AMR sensor is given by

$U_{\text {sensor }}=R_{\text {sensor }} \cdot I+U_{C}(t)$

where I is the constant current through the AMR resistor and

$U_{C}(t)=\hat{U}_{C} \sin \left(\omega_{\mathrm{exc}} t\right)$

is the capacitive coupling voltage induced by the coil layer. The spectrum of the output voltage can be seen in Fig. 2.

To isolate the needed signal a common superhet structure can be used consisting of a band-pass filter, a mixer and a 


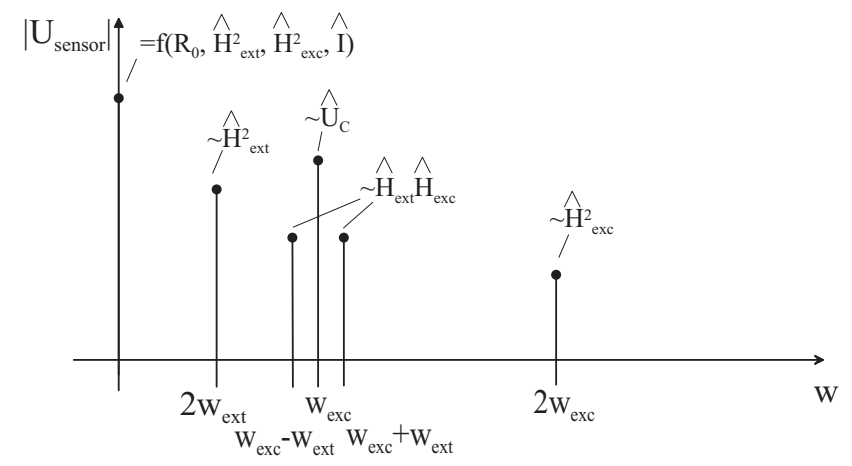

Fig. 2. Spectrum of the output voltage $U_{\text {sensor }}$ with constant current through the AMR resistor.

low-pass filter (Meade, 1994). In comparison with conventional smart sensor systems this method needs three further components: two filters and a mixer circuit, which increase costs of the signal processing unit. To reduce the needed chip area of the filter arrangement, a higher excitation frequency seems to be useful. However, there is a capacitive coupling between the on-chip coil and the AMR layer, which depends on the modulation frequency. Especially in the case of a higher excitation frequency an additional offset appears so that the advantages of AC-biasing are eliminated. To produce relief we will show a methodology, which drastically reduces the needed filters and eliminates the must of the mixer circuit. Moreover, by means of this approach capacitive coupling is eliminated by the method itself.

If we perform the mixing by means of Ohm's law, i.e. we choose an adequate sinusoidal current through the AMR resistors (see Fig. 1) with

$I(t)=\hat{I} \sin \left(\omega_{\mathrm{exc}} t\right)$

it follows

$$
\begin{aligned}
& U_{\text {sensor }}=\left[R_{0}+\Delta R\left(1-\frac{\hat{H}_{\mathrm{ext}}^{2}}{2 H_{0}^{2}}-\frac{\hat{H}_{\mathrm{exc}}^{2}}{2 H_{0}^{2}}\right)\right] \cdot \ldots \\
& \cdot \hat{I} \sin \left(\omega_{\mathrm{exc}} t\right)+\Delta R \frac{\hat{H}_{\mathrm{ext}}^{2}}{4 H_{0}^{2}} \cdot \hat{I}\left[\sin \left(\left(\omega_{\mathrm{exc}}-2 \omega_{\mathrm{ext}}\right) t\right)+\cdots\right. \\
& \left.\cdots+\sin \left(\left(\omega_{\mathrm{exc}}+2 \omega_{\mathrm{ext}}\right) t\right)\right]+\cdots \\
& \cdots+\Delta R \frac{\hat{H}_{\mathrm{ext}}^{2}}{4 H_{0}^{2}} \cdot \hat{I}\left[\sin \left(3 \omega_{\mathrm{exc}} t\right)-\sin \left(\omega_{\mathrm{exc}} t\right)\right]-\cdots \\
& \cdots-\Delta R \frac{\hat{H}_{\mathrm{ext}}^{2} \cdot \hat{H}_{\mathrm{exc}}^{2}}{2 H_{0}^{2}} \cdot \hat{I}\left[2 \sin \left(\omega_{\mathrm{ext}} t\right)+\cdots\right. \\
& \left.\cdots+\sin \left(\left(2 \omega_{\mathrm{exc}}-\omega_{\mathrm{ext}}\right) t\right)-\sin \left(\left(2 \omega_{\mathrm{exc}}+\omega_{\mathrm{ext}}\right) t\right)\right]+\cdots \\
& \cdots+\hat{U}_{C} \sin \left(\omega_{\mathrm{exc}} t\right) .
\end{aligned}
$$

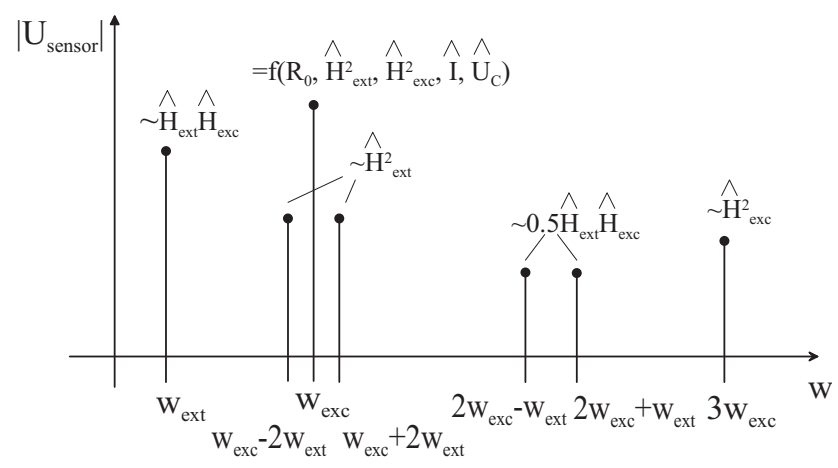

Fig. 3. Spectrum of the output voltage $U_{\text {sensor }}$ with sinusoidal current through the AMR resistor.

The spectrum of the output signal is shown in Fig. 3. To isolate the needed signal at $\omega_{\text {ext }}$ a simple low pass filter can be used. It turns out that by means of the modulation of the AMR elements the capacitive effects are separated, offset effects are eliminated and consequently temperature and life time drift are significantly reduced. In addition, since the wanted signal is offset free it can be amplified strongly. For this reason the sensitivity can be controlled directly by the amplification which makes large sensing distances possible. On this account external fields down to sensor noise can failsafe detected. Moreover, since in contrast to Barber pole sensors a bias magnet is not needed, the sensitivity is improved, where the production costs are reduced. However, the low pass filter has high requirements to exfiltrate higher frequencies without a phase shift of the output signal.

To reduce the requirements of the filter one can use a difference principle desribed below. The initial point is Eq. (5)

$$
\begin{gathered}
R_{\mathrm{sensor}}^{+}(t)=R_{0}+\Delta R-\Delta R \frac{\hat{H}_{\mathrm{ext}}^{2}}{H_{0}^{2}} \sin ^{2}\left(\omega_{\mathrm{ext}} t\right)-\cdots \\
\cdots-2 \Delta R \frac{\hat{H}_{\mathrm{ext}} \hat{H}_{\mathrm{exc}}}{H_{0}^{2}} \sin \left(\omega_{\mathrm{ext}} t\right) \sin \left(\omega_{\mathrm{exc}} t\right)-\cdots \\
\cdots-\Delta R \frac{\hat{H}_{\mathrm{exc}}^{2}}{H_{0}^{2}} \sin ^{2}\left(\omega_{\mathrm{exc}} t\right),
\end{gathered}
$$

where $H_{y}$ is

$H_{y}=\hat{H}_{\text {ext }} \sin \left(\omega_{\text {ext }} t\right)+\hat{H}_{\text {exc }} \sin \left(\omega_{\text {exc }} t\right)$.

If we excite a second comparable AMR element with

$$
H_{y}=\hat{H}_{\text {ext }} \sin \left(\omega_{\text {ext }} t\right)-\hat{H}_{\text {exc }} \sin \left(\omega_{\text {exc }} t\right)
$$

we arrive at:

$$
\begin{gathered}
R_{\mathrm{sensor}}^{-}(t)=R_{0}+\Delta R-\Delta R \frac{\hat{H}_{\mathrm{ext}}^{2}}{H_{0}^{2}} \sin ^{2}\left(\omega_{\mathrm{ext}} t\right)+\cdots \\
\cdots+2 \Delta R \frac{\hat{H}_{\mathrm{ext}} \hat{H}_{\mathrm{exc}}}{H_{0}^{2}} \sin \left(\omega_{\mathrm{ext}} t\right) \sin \left(\omega_{\mathrm{exc}} t\right)-\cdots
\end{gathered}
$$




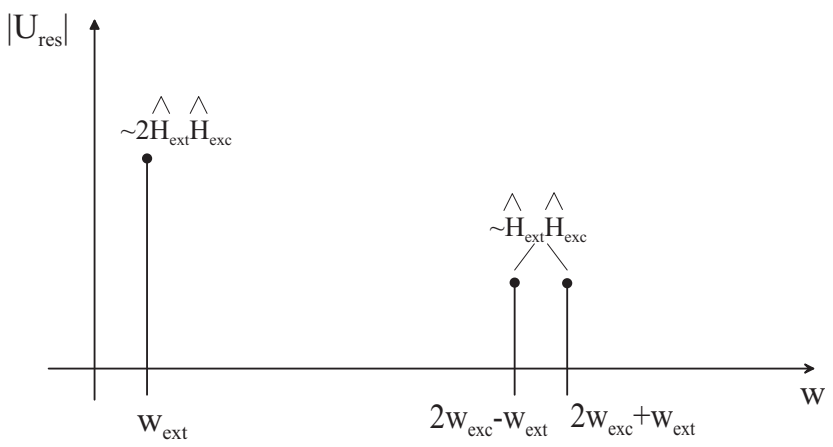

Fig. 4. Spectrum of the resulting output voltage $U_{\text {res }}$ by using the difference principle.

$$
\cdots-\Delta R \frac{\hat{H}_{\mathrm{exc}}^{2}}{H_{0}^{2}} \sin ^{2}\left(\omega_{\mathrm{exc}} t\right)
$$

The resulting output voltage

$$
\begin{aligned}
& U_{\text {res }}=U_{\text {sensor } 1}+U_{\text {sensor } 2} \\
& =R_{\text {sensor }}^{+}(t) \cdot \hat{I} \sin \left(\omega_{\text {exc }} t\right)+\hat{U}_{C} \sin \left(\omega_{\mathrm{exc}} t\right)+\cdots \\
& \cdots+R_{\text {sensor }}^{-}(t) \cdot \hat{I} \sin \left(-\omega_{\mathrm{exc}} t\right)+\hat{U}_{C} \sin \left(-\omega_{\mathrm{exc}} t\right) \\
& =-\Delta R \frac{\hat{H}_{\mathrm{ext}} \hat{H}_{\mathrm{exc}}}{H_{0}^{2}} \hat{I} \cdot\left[2 \sin \left(\omega_{\mathrm{ext}} t\right)+\cdots\right. \\
& \left.\cdots+\sin \left(\left(2 \omega_{\mathrm{exc}}-\omega_{\mathrm{ext}}\right) t\right)+\sin \left(\left(2 \omega_{\mathrm{exc}}+\omega_{\mathrm{ext}}\right) t\right)\right]
\end{aligned}
$$

represents the wanted speed signal and components around the double excitation frequency. These higher frequencies can easily be filtered by a low pass with low order (see Fig. 4). The complete sensor concept is shown in Fig. 5. By means of this invention the conversion of the speed signal from bandpass to baseband takes place in the AMR element itself and needs no mixer. Furthermore, the disturbing capacitive coupled signal is eliminated by this new approach and the requirements of the filter are reduced by the difference principle. Therefore, the invention is based on a simple low pass filter with low order and high edge frequency preventing frequency dependent phase shift between magnetic input and electric output signal. In addition, the presented structure fulfils low cost requirements concerning the signal conditioning ASIC.

If the amplitude of the magnetic input signal is large - this means that the magnetic input signal fulfils $\left|H_{y}\right|>H_{0}$ and the transfer function loses its quadratic characteristic - higher harmonics can appear. It is impractical to separate these perturbations from the wanted signal by filter operations. On this account a traditional concept which is based on a superhet structure is not able to measure magnetic signal with amplitudes larger than $H_{0}$. In contrast, as shown above the suggested sensor concept suppresses signals which are not

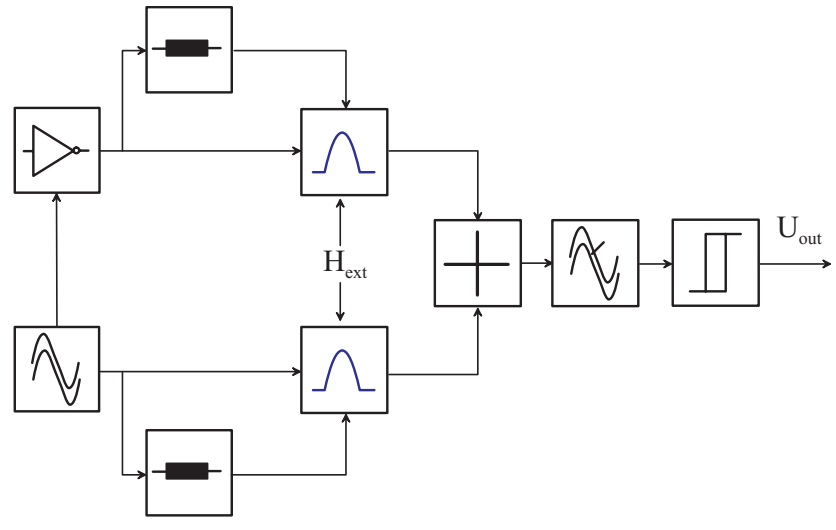

Fig. 5. Block diagram of the signal processing unit.

in phase with the wanted signal. So this structure is able to detect magnetic inputs with $\left|H_{y}\right|>H_{0}$. If we assume

$$
\begin{aligned}
H_{y} & =\hat{H}_{\mathrm{ext}} \sin \left(\omega_{\mathrm{ext}} t\right)+\hat{H}_{\mathrm{exc}} \sin \left(\omega_{\mathrm{exc}} t\right) \\
& \approx \hat{H}_{\mathrm{ext}} \sin \left(\omega_{\mathrm{ext}} t\right)
\end{aligned}
$$

for

$\hat{H}_{\text {ext }} \gg \hat{H}_{\text {exc }}$,

we are able to calculate by using Eqs. (3) and (16) the Fourier series of the resulting resistance $R_{\text {sensor }}(t)$ for

$\hat{H}_{\text {ext }}>H_{0}$.

From this follows

$$
\begin{aligned}
& R_{\text {sensor }}(t)=R_{0}-\frac{K}{\pi}\left(\arcsin (P)-P \cdot \sqrt{1-P^{2}}\right)+\cdots \\
& +\sum_{n=1}^{\infty} \frac{K}{\pi} \cdot\left[\frac{4}{2 n} \sin (2 n \arcsin (P))-\cdots\right. \\
& -\frac{2}{2 n-2} \sin ((2 n-2) \arcsin (P))-\cdots \\
& \left.-\frac{2}{2 n+2} \sin ((2 n+2) \arcsin (P))\right] \cdot \cos \left(2 n \omega_{\mathrm{ext}} t\right)
\end{aligned}
$$

where

$K=\Delta R \frac{\hat{H}_{\mathrm{ext}}^{2}}{H_{0}^{2}}$

and

$P=\frac{H_{0}}{\hat{H}_{\text {ext }}}$.

From Eq. (19) follows that high amplitudes of the external signal do not thwart the basic properties of this principle. However, if Eq. (17) is valid, higher harmonics of $H_{\text {exc }}$ can change the amplitude of the mixed signal so that quantitative measurements are hindered. On the other hand often qualitative field sensing is sufficient, i.g. in the field of wheel speed sensing. 


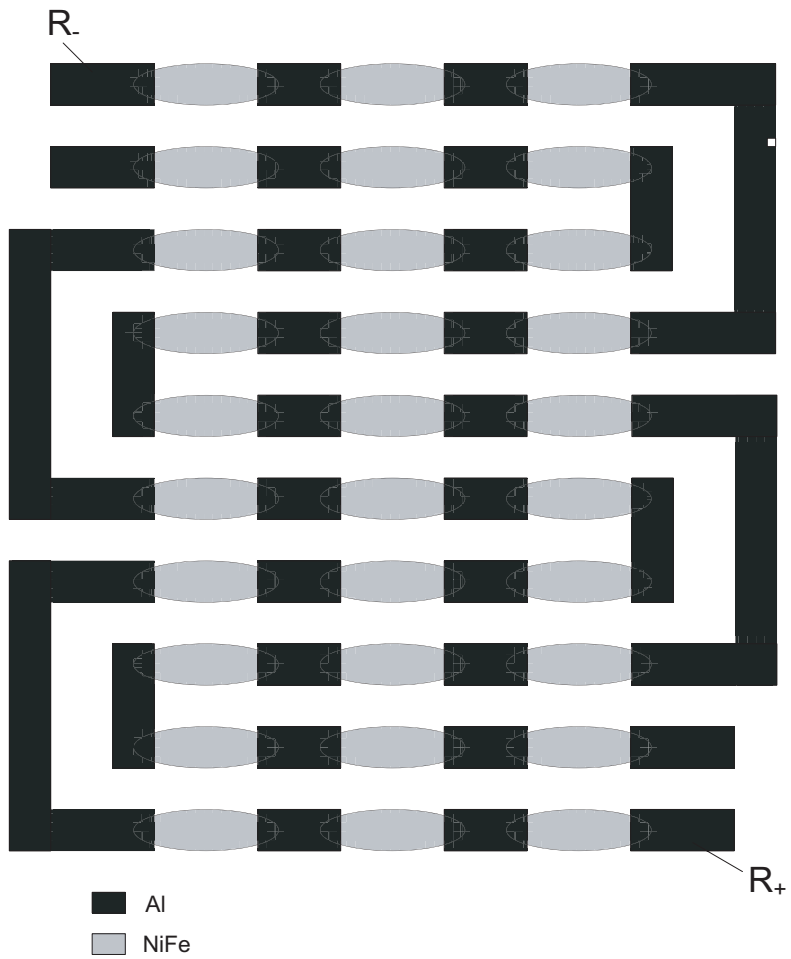

Fig. 6. Layout of the AMR layer.

\section{Experimental results}

To verify the presented methodology integrated prototypes were implemented. The signal processing units are realized according to Fig. 5. The layout of the sensing elements is similar to the KMZ51 earth field sensor (Stork, 1997). Since the methodology is based on a nonlinear transfer characteristic of the AMR elements, in contrast to the KMZ51 Barber poles were not implemented. Furthermore, only one drivecoil along the easy axis was realized to excite the sensing elements. As a matter of principle offset effects are eliminated and so temperature and life time drift are also essentially reduced. Instead of meander patterned sensor (Ciureanu, 1992), this system consists of two strips of 36 single resistors, which are not arranged in a Whetstone bridge. This leads to a further cost down, since on chip trimming of the bridge resistors is not needed. To guarantee as low as possible tolerances a sensor layout as shown in Fig. 6 was realized. The resistance of each stripe of sensing elements is approximately $14 \mathrm{k} \Omega$, while the drive coil resistance is about $200 \Omega$ and the coil field factor is approximately $25 \frac{(A / m)}{(m A)}$. So by means of relatively small excitation current through the on-chip coils a high sensitivity $S$ can be achieved, i.e. $S>20 \frac{(m V / V)}{(k A / m)}$ for $H_{x} \leq 50 \frac{A}{m}$ and $\hat{H}_{\mathrm{exc}}>100 \frac{A}{m}$.

The spectrum of the output signal of one stripe of sensing elements near the excitation signal is shown in Fig. 7, where $\omega_{\mathrm{ext}}=20 \mathrm{kHz}$ and $\omega_{\mathrm{exc}}=1 \mathrm{MHz}$. For the generation of

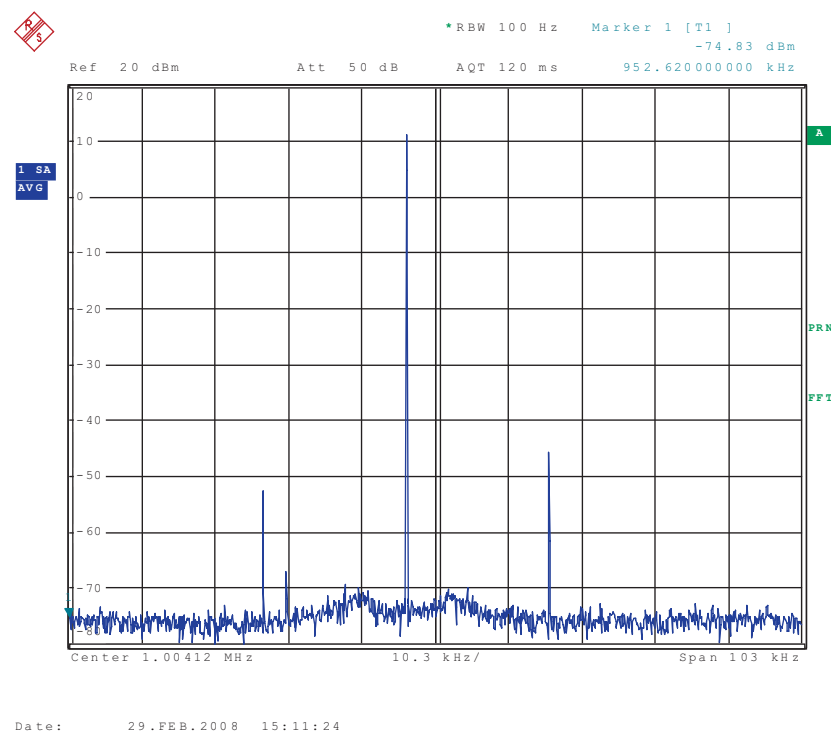

Fig. 7. The spectrum of the AMR element series $R^{+}$near $f_{\text {exc }}$.

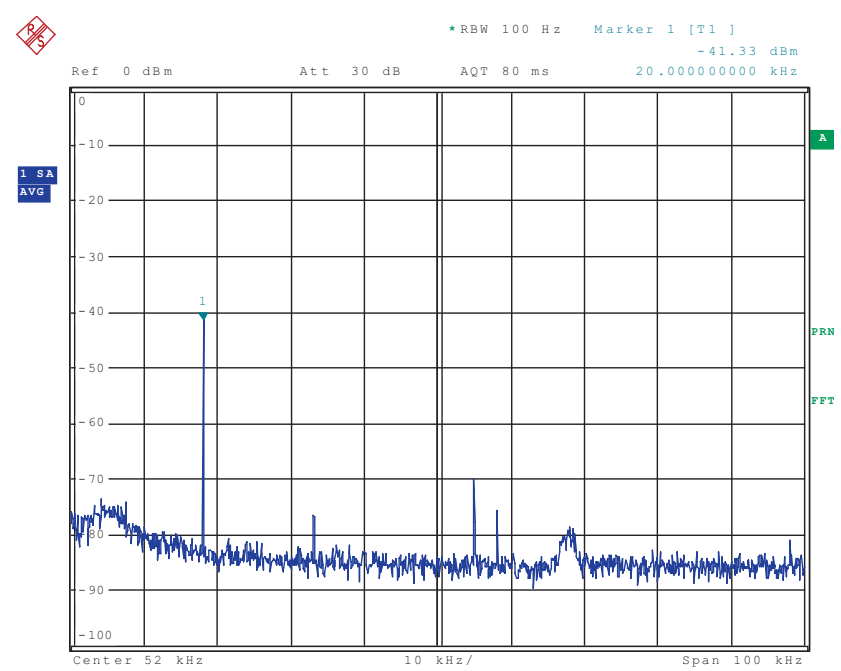

Fig. 8. The spectrum of the output signal of the smart sensor system.

the external field a Helmholz coil was used, while the ACexcitation of AMR elements was produced by current-fed onchip drive-coils. The current through the coils is to be chosen to $5 \mathrm{~mA} \cdot \sin (2 \pi 1 \mathrm{MHz} \cdot t)$. As expected there is a large spectral line at $f_{\text {exc }}$ which is a result of the capacitive coupling between the AMR and coil-layer. However, if we use the presented methodology the wanted signal is directly mixed to the base-band (see Fig. 8). Following the difference principle shown in Fig. 5 the output signals of both AMR elements are added. The remaining, undesired spectral lines can easily be removed by a simple low-pass filter. The required chip area depends on the modulation frequency. For 
a higher modulation frequency the low-pass filter is smaller and the phase shift of the sensor output signal compared to $H_{\text {ext }}(t)$ is lower. Further investigations show that in contrast to common used weak field sensors both techniques are robust against large external fields. So in case of constant magnetic fields the measurement range in $\pm H_{0}$, while periodical signals with amplitudes of up to $6 H_{0}$ can be detected.

\section{Conclusions}

In this paper a novel method for weak field sensing is presented. The highly integrated prototype demonstrates feasibility and outstanding performance with respect to the requirements in industrial and especially automotive applications such as low cost, low power and high robustness. The sensor system is able to detect fields in the order of the sensor noise and possess a large measurement range. As a matter of principle offset effects are eliminated and on this account temperature and life time drift are essentially reduced. Since trimming of bridge resistors as well as a bias magnet is not needed the production costs are essentially decreased. Summarizing, performance and cost efficiency are outstanding in comparison to each commercial available magnetic sensor system and justify the relevance of AMR technology for industrial and especially automotive applications.

\section{References}

Ciureanu, P.: Magnetoresistive Sensors, in: Thin film resistive sensors, edited by: Ciureanu, P. and Middelhoek, P., Institute of Physics Publishing, 253-436, 1992.

de Niet, E. and Vreeken, R.: A magnetoresistive head with magnetic feedback, IEEE T. Magn., 15(6), 1625-1628, September 1979.
Dibbern, U.: Magnetoresistive Sensors, in: Sensors - A Comprehensive Survey, edited by: Göpel, W., Hesse, J., and Zemel, J. N., Vol. 5, Magnetic Sensors, edited by: Boll, R. and Overshott, K. J., Wiley-VCH, Weinheim, 342-379, 1989.

Flynn, D.: Demodulation of a magnetoresistive sensor signal to achieve a low-cost, stable and high-resolution vector magnetometer, Elsevier Science, Sensor. Actuat. A-Phys., 50, 187-190, 1995.

Kuijk, K. E., van Gestel, W. J., and Gorter, F. W.: The Barber Pole, a linear magnetoresistive head, IEEE Trans. Magn., 11(5), 12151217, September 1975

Meade, M. L.: Lock-in amplifiers: principles and applications, IEE Series in Measurement, Peregrinus, 5-22, 1994.

Petrou, J., Dimitropoulos, P. D., Hristoforou, E., and Neagu, N.: New 2D fluxgate device based on the phase modulation of magnetization rotation in AMR films, Elsevier Science, Sensor. Actuat. A-Phys., 129, 107-111, 2006.

Razavi, B.: RF Microelectronics, Prentice Hall, 54-96, 1997.

Ripka, P.: Magnetic Sensors and Magnetometers, Arttech House, 130-150, 2000.

Ripka, P., Tondra, M., Stokesa, J., and Beech, R.: AC-driven AMR and GMR magnetoresistors, Elsevier Science, Sensor. Actuat. APhys., 76(1), 225-230, August 1999.

Stork, T.: Electronic Compass Design using KMZ51 and KMZ52, Application Note AN00022, NXP Semiconductors, 1997.

Tumanski, S.: A new Type of Thin Film Magnetoresistive Magnetometers - an Analysis of Circuit Principles, IEEE T. Magn., 20(5), 1720-1722, September 1984.

Tumanski, S.: Thin Film Magnetoresistive Sensors, IOP Publishing, 33-145, 2001.

Ueda, M., Endoh, M., Yoda, H., and Wakatsuki, N.: AC Bias Type Magnetoresitive Sensor, IEEE T. Magn., 26(5), 1572-1574, 1999. 